\title{
The effect of eating on the uptake of PSMA ligands in the salivary glands
}

\author{
V. Mohan ${ }^{1,2}$, N N. M. Bruin ${ }^{1,2}$, J. B. van de Kamer², J.-J. Sonke² and W. V. Vogel ${ }^{1,2^{*}}$
}

\begin{abstract}
Rationale: PSMA-directed therapy for metastatic prostate cancer is gaining adoption as a treatment option. However, accumulation of ${ }^{177} \mathrm{Lu} /{ }^{225} \mathrm{Ac}-\mathrm{PSMA}$ in the salivary glands remains a problem, with risk of dose-limiting xerostomia and potentially severe effect on the quality of life. Gustatory stimulation is an approach that has commonly been used in radioactive iodine therapy to reduce accumulation in the salivary glands. However, based on theoretical differences in biodistribution, it was hypothesized that this could potentially lead to adverse increased toxicity for PSMA-ligand therapy. The primary objective of this work was to determine if gustatory stimulation by eating an assortment of sweet/fatty/acidic foods during the biodistribution phase of $\left[{ }^{18} \mathrm{~F}\right] \mathrm{DCFPyl}$ could result in a clinically relevant (>30\%) change in the uptake of the tracer in the salivary glands.

Methods: 10 patients who already received a whole-body $\left[{ }^{18} \mathrm{~F}\right]$ DCFPyI PET/CT scan for evaluation of prostate cancer, underwent a repeat (intervention) PET/CT scan within a month of the first (control) scan. During the intervention scan, patients chose from an assortment of sweet/fatty/acidic foods, which they then chewed and swallowed for a period of time starting $1 \mathrm{~min}$ before tracer administration to $10 \mathrm{~min}$ thereafter. Data from both scans were analyzed by placing VOIs on the major salivary glands and segmenting them using relative thresholds.

Results: A slight increase in PSMA uptake in the parotid glands was observed on the intervention scan when compared to the baseline scan $\left(+7.1 \% \mathrm{SUL}_{\text {mean }}\right.$ and $\left.+9.2 \% \mathrm{SUL}_{\max }, p<0.05\right)$. No significant difference in PSMA uptake in the submandibular glands was seen.
\end{abstract}

Conclusions: Eating only slightly increases uptake of $\left[{ }^{18} \mathrm{~F}\right] \mathrm{DCFPyl}$ in the parotid glands. We nonetheless recommend refraining from gustatory stimulation during the administration and early biodistribution phase of radionuclide therapy with PSMA-ligands to reduce the risk of avoidable additional toxicity.

Keywords: Salivary glands, Toxicity, PSMA, Radionuclide therapy, Stimulation

\section{Introduction}

Radioligand therapy (RLT) with prostate specific membrane antigen (PSMA) ligands has been gaining adoption as a treatment option for patients with metastatic prostate cancer. While the PSMA receptor is overexpressed in prostate cancer tissue, it is also expressed in certain normal tissues, such as the salivary glands [1-3]. As a result, PSMA-ligands tend to accumulate in these glands

\footnotetext{
*Correspondence: w.vogel@nki.nl

${ }^{1}$ Department of Nuclear Medicine, The Netherlands Cancer Institute, Plesmanlaan 121, 1066 CX Amsterdam, The Netherlands

Full list of author information is available at the end of the article
}

during therapy, although a large fraction of this has been attributed to an unknown, non-specific mechanism that is unassociated with receptor expression $[4,5]$. Regardless, this uptake during therapy is undesirable, and can potentially result in radiation induced toxicity, manifesting as xerostomia (dry mouth syndrome). Xerostomia from $\left[{ }^{177} \mathrm{Lu}\right] \mathrm{Lu}-\mathrm{PSMA}-617$ therapy is reportedly limited [6], but since it is administered over an increasing number cycles, the risk of toxicity is cumulative. In the case of alpha emitting $\left[{ }^{225} \mathrm{Ac}\right]$ Ac-PSMA-617 therapy, which has shown promise beyond [ $\left.{ }^{177} \mathrm{Lu}\right] \mathrm{Lu}-\mathrm{PSMA}-617$ therapy in some cases [7], xerostomia is frequent and can be severe enough to warrant patients discontinuing treatment [8]. 
Thus, there is a growing need to develop strategies to mitigate this potentially dose-limiting toxicity, if PSMA therapy is to see widespread clinical use.

A strategy that is frequently employed in radioactive iodine $\left({ }^{131} \mathrm{I}\right)$ therapy for differentiated thyroid cancer to reduce salivary gland toxicity, is gustatory stimulation [9]. Salivary gland cells express the sodium iodide symporter (NIS), which leads to accumulation of ${ }^{131}$ I within the gland [10]. Iodine taken up by the salivary glands is not organified as it is in the thyroid. Thus, by stimulating the secretion of saliva with a sialagogue like lemon juice, the residence or transit time of ${ }^{131}$ I within the gland is shortened, as it is excreted or washed out [11]. On the other hand, this stimulation also leads to an increase in blood flow to the glands [12], and given their extensively perfused vasculature, this could result in an undesirable increase in the delivery of ${ }^{131}$ I to the glands. This is sometimes referred to as the 'rebound effect', and its impact is disputed [13].

Gustatory stimulation has not been tested in the context of PSMA-ligands before. It could either increase tracer uptake due to increased perfusion, or reduce it by increasing excretion. Both potential effects on (specific and/or non-specific) PSMA uptake represent important knowledge that can contribute toward optimising guidelines for diagnostic procedures or therapy.

Positron emission tomography (PET) with diagnostic PSMA-ligands like $\left[{ }^{68} \mathrm{Ga}\right] \mathrm{Ga}$-PSMA-11 or $\left[{ }^{18} \mathrm{~F}\right] \mathrm{DCFPyl}$ (a radiofluorinated PSMA inhibitor) allows for screening the effects of such strategies, without the consequences that any unforeseen changes in biodistribution would have on therapy. In this study, we examined the effect of gustatory stimulation by eating during the initial administration and biodistribution phase, on the uptake of $\left[{ }^{18} \mathrm{~F}\right]$ DCFPyl in the salivary glands using intra-patient controlintervention PET scans. A change of more than $30 \%$ of the baseline was considered clinically relevant.

\section{Methods}

The Medical Ethics Committee of the Netherlands Cancer Institute (CCMO trial registration NL71902.031.20) approved the study protocol. The study was conducted in accordance with the Declaration of HELSINKI. Written and oral informed consent was obtained from all patients prior to study entry.

\section{Study population}

The study included 10 patients with prostate cancer, who recently $(<1$ month ago $)$ received a $\left[{ }^{18} \mathrm{~F}\right] \mathrm{DCFPyl} \mathrm{PET} /$ CT whole-body scan for evaluation of prostate cancer on clinical indication, wherein the salivary glands were clearly visible. This first scan served as the baseline or control scan. Patients with a history of disease or treatment involving the salivary glands were excluded. Patients who received any treatments since the control scan was acquired were excluded.

\section{Study procedure and image acquisition}

On the days of both the control and intervention scans, patients were pre-hydrated with $0.5 \mathrm{~L}$ of water and did not consume food shortly before tracer administration. For the control scan, patients were injected intravenously with $200 \mathrm{MBq}$ of $\left[{ }^{18} \mathrm{~F}\right] \mathrm{DCFPyl}$ (good manufacturing practice grade, produced by BV Cyclotron VU, The Netherlands). After an incubation time of $45-60 \mathrm{~min}$, patients were scanned from the upper thighs to the base of the skull using a digital PET/CT scanner (Vereos, Philips Healthcare, The Netherlands). A low dose CT scan was acquired with $120 \mathrm{kV}, 30 \mathrm{mAs}$ and $2 \mathrm{~mm}$ slices. PET images were acquired with 2 min per bed position. All scans were reconstructed iteratively using the ordered subset estimation maximization algorithm (3 iterations and 15 subsets) to $4 \times 4 \times 4 \mathrm{~mm}^{3}$ voxels, with a gaussian filter full width-half maximum of $3 \mathrm{~mm}$ and with attenuation correction.

The intervention PET scan was performed within a month of the control scan. On the day of the intervention scan, patients were offered a selection of salivary stimulating food items rich in sugars, acids or fats, such as caramel, fudge, sour candy, chocolate cookies, liquorice and nougat. Patients were asked to chew and swallow the food items of their preference for a period starting $1 \mathrm{~min}$ prior to injection of $\left[{ }^{18} \mathrm{~F}\right] \mathrm{DCFPyl}$, until $10 \mathrm{~min}$ after injection. The remainder of the imaging procedure for the intervention scan was identical to that of the control scan, and so were the acquisition and reconstruction parameters.

\section{Image analysis}

Uptake on the PET scans was measured quantitatively using in-house developed software. Standardised uptake values corrected for lean body mass (SUL) were calculated using James' formula [14]. Four 3D volumes of interest (VOI) were initially placed around each of the parotid and submandibular glands on each scan. A relative threshold of $20 \%$ of the maximum uptake value within each VOI was used to segment them. $\mathrm{SUL}_{\text {mean }}$ and $\mathrm{SUL}_{\max }$ for each of the salivary glands were measured. Additionally, the liver and aorta were included as reference organs. $\mathrm{SUL}_{\text {mean }}$ for these organs was measured by placing a spherical VOI of 3-cm-diameter [15] in the same representative reference location in both scans.

\section{Statistical analysis}

Statistical analysis was done using R v4.0.4 [16]. For the salivary glands, a linear mixed effects analysis of the 
relationship between uptake parameters and stimulation was performed using the lme 4 package [17]. This was done to account for multiple measurements per gland type (left and right) from individual patients. Stimulation (control or intervention scan) and laterality (left or right) were entered as fixed effects without any interaction. Individual intercepts for each patient were fit as random effects. Log likelihood ratio tests were used to arrive at p-values, by comparing the full model to a reduced model without the stimulation fixed effect. Comparisons between the control and intervention uptake for the reference organs were done using a two-tailed paired samples t-test, after checking for normality according to the Shapiro-Wilk test. Significance level of $\alpha=0.05$ was selected in all tests.

\section{Results}

\section{Demographics}

The 10 included male patients had an average age of 66 years (range of 48-75). On the days of the intervention scan, patients selected from an assortment of saliva stimulating foods and consumed on average $116 \mathrm{~g}$ (range of 60-200 g) over the course of $11 \mathrm{~min}$.

\section{$\mathrm{PET} / \mathrm{CT}$ data}

Representative control and intervention scans are shown in Fig. 1. Uncropped total body scans can be seen in Additional file 1: Supplemental Fig. 1. The mean \pm standard deviation of various SUL parameters for each of the different tissue types investigated in all patients, along with the relative change (and 95\% confidence intervals in squared brackets) are shown in Table 1. Salivary gland data in Table 1 are averaged laterally. Upon visual inspection of the salivary gland data, there were no obvious deviations from the assumptions of normality and homoscedasticity. The reference organs also passed normality test criteria. No significant differences between the control and intervention SUL parameters were found for the submandibular glands and the liver. In the parotid glands, a small increase in $\mathrm{SUL}_{\text {mean }}(7.1 \%, p=0.023)$ and $\mathrm{SUL}_{\max }$ $(9.2 \%, p=0.016)$ was observed and was statistically significant when controlling for random patient effects, though this was lower than the predefined threshold for clinical relevance (Fig. 2). A small but significant reduction in $\mathrm{SUL}_{\text {mean }}$ in the aorta was also observed $(6.0 \%$, $p=0.003$ ). Plots for other tissues and SUL paramters can be seen in Additional file 1: Supplemental Fig. 2-6.

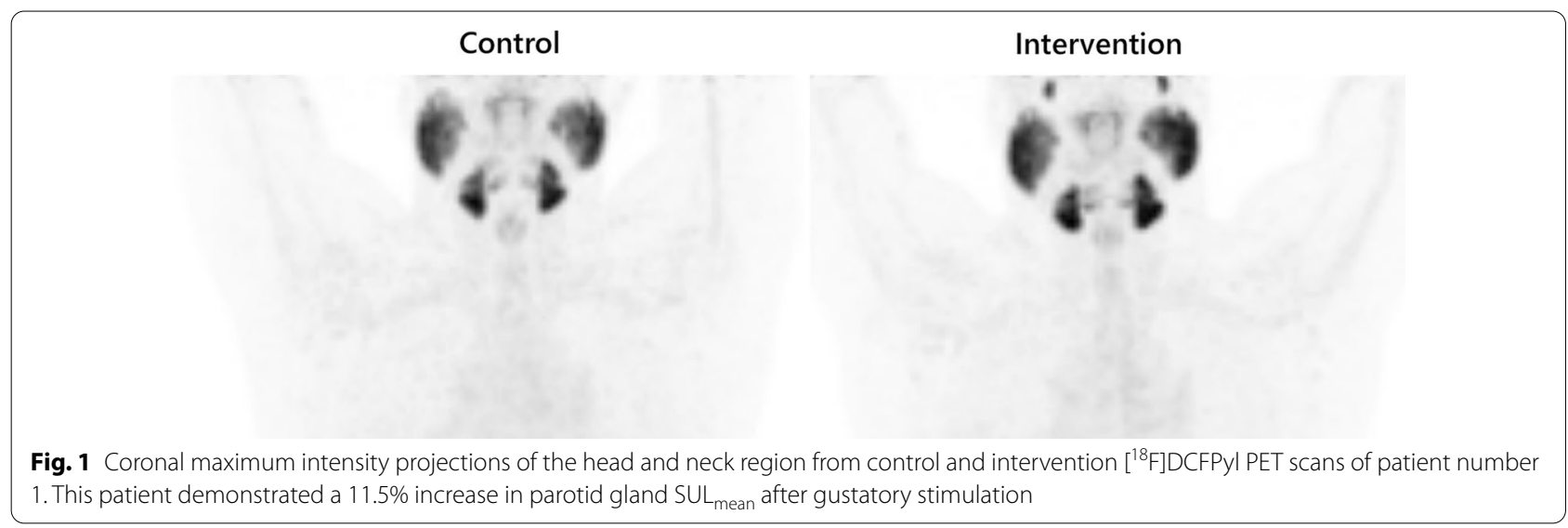

Table 1 Comparison of $\left[{ }^{18} \mathrm{~F}\right]$ DCFPyl PET parameters in tissues for control and intervention scans

\begin{tabular}{llcccc}
\hline Tissue & Parameter & \multicolumn{1}{c}{ Control } & Intervention & Relative change (\%) & $\boldsymbol{p}$ Value \\
\hline Parotid glands & $S U L_{\text {mean }}$ & $6.04 \pm 1.29$ & $6.47 \pm 1.82$ & $7.1[1.1,13.2]$ & $0.023^{*}$ \\
& $S U L_{\text {max }}$ & $12.29 \pm 2.70$ & $13.43 \pm 3.80$ & $9.2[1.9,16.6]$ & $0.016^{*}$ \\
Submandibular glands & $S U L_{\text {mean }}$ & $6.16 \pm 1.62$ & $6.28 \pm 2.29$ & $2.1[-5.8,9.9]$ & 0.606 \\
& $S U L_{\text {max }}$ & $12.76 \pm 3.16$ & $12.99 \pm 4.37$ & $1.8[-6.6,10.2]$ & 0.667 \\
Aorta & $S U L_{\text {mean }}$ & $1.14 \pm 0.13$ & $1.07 \pm 0.15$ & $-6.0[-9.4,-2.6]$ & $0.003^{*}$ \\
Liver & $S U L_{\text {mean }}$ & $3.95 \pm 0.77$ & $3.79 \pm 0.57$ & $-3.9[-15.1,7.2]$ & 0.446 \\
\hline
\end{tabular}

SUL, Standardised uptake value corrected for lean body mass corrected

${ }^{*} p<0.05$ is considered statistically significant 


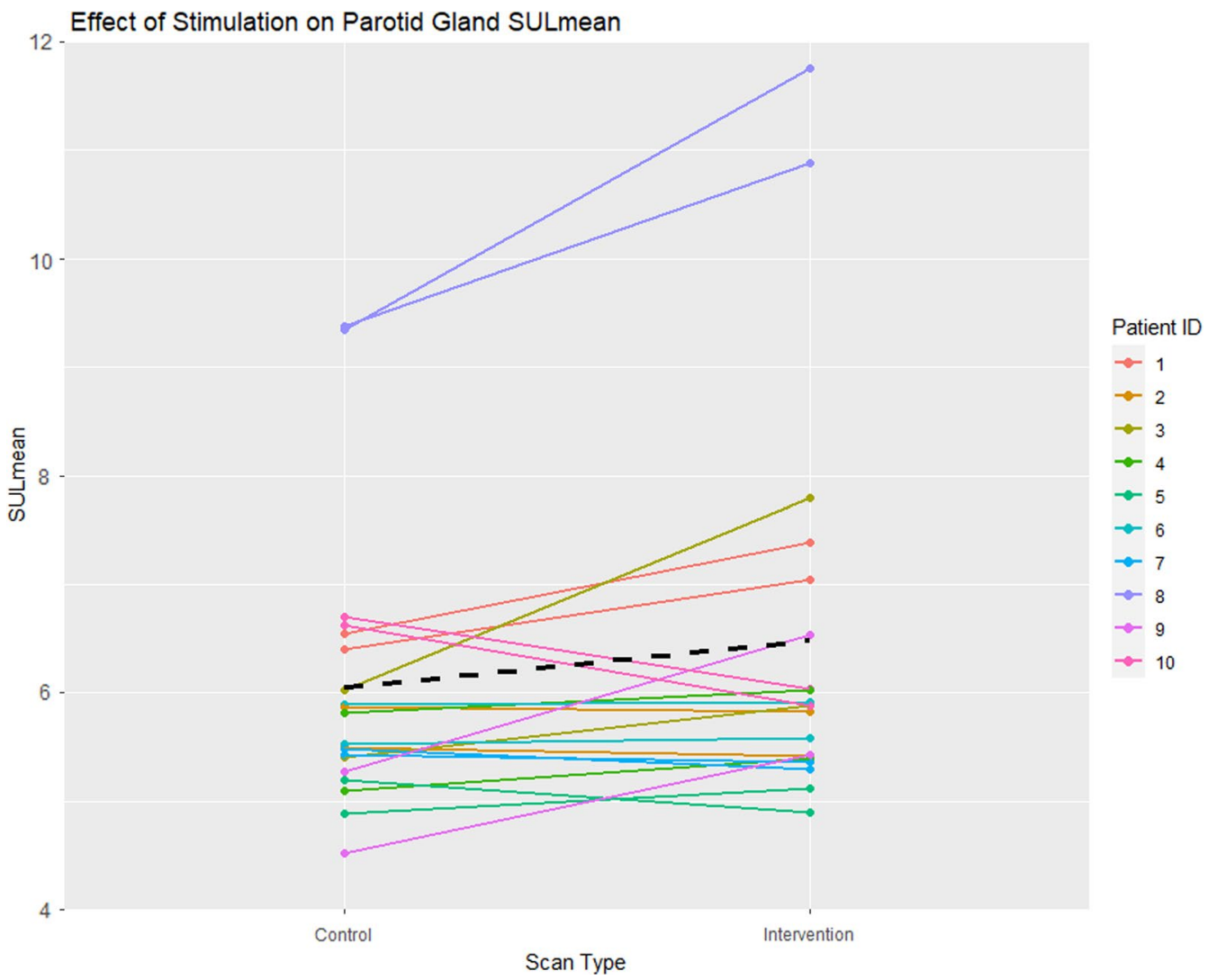

Fig. 2 Effect of gustatory stimulation on parotid gland $S U L_{\text {mean. }}$. Each patient is represented by a unique colour. The patients have two lines, one for the right parotid and one for the left parotid. The black dashed line depicts the overall population effect for all parotid glands of all patients

\section{Discussion}

This study evaluated the effects of gustatory stimulation by eating on the uptake of the PSMA-ligand $\left[{ }^{18} \mathrm{~F}\right] \mathrm{DCFPyl}$ in salivary glands and found that it resulted in a limited increase. Despite the effect size falling short of our initial definition of clinical relevance, we believe that this is sufficient to warrant a recommendation against gustatory stimulation or eating during the administration of PSMA targeted RLTs.

\section{Gustatory stimulation in ${ }^{131} \mid$ therapy}

The effectiveness of gustatory stimulation, as well as the timing of it, in order to reduce the absorbed dose to salivary glands from ${ }^{131}$ I therapy is still under debate. Various studies involving non-pharmacological sialagogues like lemon juice/slices/candy, vitamin $\mathrm{C}$ and tasteless chewing gum have been performed using dynamic ${ }^{99 \mathrm{~m}} \mathrm{Tc}$ and ${ }^{123}$ I scintigraphy scans, as well as multiple-time-point ${ }^{124} \mathrm{I}$ PET/CT scans [18]. These studies have yielded contradictory results [19], with some hypothesizing a decrease in absorbed dose due to excretion [20,21], some an increase due to blood flow [22], and others questioning any effect whatsoever [23, 24]. Furthermore, there is contention over whether the sialagogue in question should be given immediately after administration of ${ }^{131}$ I, or 24 h later [19, 21]. Nonetheless, stimulation with lemon juice at either time point is recommended in guidelines for ${ }^{131}$ I therapy [9].

\section{Translation to PSMA}

Unlike iodine, PSMA-ligands that are accumulated in the salivary glands stay bound. PSMA receptors are expressed constitutively in salivary gland tissue, and a portion of the uptake is bound to these receptors. PSMA is internalized in tumours [25] and if this is also true of salivary glands, we would expect stimulation to have no effect on the excretion of it out of the gland. The other portion of the uptake is believed to be bound by a nonspecific mechanism unrelated to receptor expression and may or may not be displaced by stimulation $[4,5]$. Since few strategies so far have been able to lower PSMA uptake selectively in the salivary glands without compromising tumour uptake [26], we determined that this approach was worth evaluating. However, as in the case 
of iodine, stimulation could also lead to an increase in PSMA uptake caused by an increase in blood flow.

Our study demonstrates that gustatory stimulation during the initial administration and bio-distribution phase of injected $\left[{ }^{18} \mathrm{~F}\right] \mathrm{DCFPyl}$, does indeed slightly increase (7-9\%) uptake in the parotid glands, when compared to a control. No significant difference was found in the submandibular glands. A possible explanation for this is that parotid glands are largely responsible for saliva production during stimulation [27]. A slight decrease $(6 \%)$ in uptake was found in the aorta. Whether or not this decrease may in part be related to the increase in uptake in the parotid glands is speculative and cannot be determined from our data, but may be worth investigating in another study.

Few prior studies have tested the effects of sialagogues in the context of PSMA-ligand uptake in the salivary glands. Afshar-Oromieh et al. [28] orally administered vitamin $C$ to patients every $30 \mathrm{~min}, 1 \mathrm{~h}$ after injection of [ ${ }^{68} \mathrm{Ga}$ ]Ga-PSMA-11 and found no changes in PSMA uptake on the $3 \mathrm{~h}$ post injection scan when compared to unstimulated scans from a previous dataset. The lack of a reduction, in agreement with our findings, supports the hypothesis that PSMA uptake in the salivary glands, whether specific or non-specific, cannot be displaced by stimulation. The absence of any noticeable increase is explicable by the late time point at which they administered the sialagogue. Since in our study the stimulation was immediate, an increase in blood flow would result in more unbound PSMA being delivered to the salivary glands than when compared to a stimulation at $1 \mathrm{~h}$ post injection, where most of the PSMA is already bound. PSMA-ligand concentration in arteries decays by $80 \%$ and plateaus within 5 min of tracer administration [29]. Furthermore, given that our study showed only a small effect size, it is unsurprising they found no significant differences.

The same group, in another study [30, 31], used lemon juice 5 times per day as well as ice packs on the first day of therapy in patients receiving $\left[{ }^{131} \mathrm{I}\right] \mathrm{I}-\mathrm{MIP}-1095$ (a radioiodinated PSMA inhibitor) therapy. They questioned its efficacy in lowering gland uptake, which was unverifiable due to the lack of a control group. Hypothetically, externally applied icepacks should cause vasoconstriction and reduce blood flow, thereby also decreasing PSMA delivery. If lemon juice doesn't excrete PSMA out of the gland, the increase in blood flow it produces might confound or offset any protection that ice packs may offer. However, recent studies have shown that external cooling has little to no impact on PSMA uptake [32, 33].
A limitation of our study is the low number of patients, whom were all male. Moreover, the variety and intensity of stimulatory effects that different foods can elicit, and any consequential effects on PSMA uptake were not controlled for. The results obtained are also potentially dependent on the timing at which the stimulation is given. Extrapolation of our results from diagnostic scans to a therapeutic setting may not be completely robust given potential differences in uptake and biodistribution time between diagnostic and therapeutic PSMA-ligands. We recommend that our observations be validated, preferably in another centre.

Current $\left[{ }^{177} \mathrm{Lu}\right] \mathrm{Lu}-\mathrm{PSMA}$ therapy guidelines do not recommend against eating during therapy for patients, nor do they advocate for or against the use of sialagogues [34]. They also permit for up to 6 cycles of therapy with an injected activity of $7.4 \mathrm{GBq}$ per cycle. Previous studies that reported on xerostomia rates were limited to fewer cycles [6]. This escalation of treatment by increasing the number of cycles delivered can only be achieved by minimizing the risk of intolerable toxicity. As toxicity can accumulate over cycles, even small deleterious effects can add up. Dose limits in external beam radiotherapy for parotid glands and submandibular glands have been established in the range of 26-39 Gy [35, 36]. The dose to the salivary glands from a $7.4 \mathrm{GBq}$ cycle of $\left[{ }^{177} \mathrm{Lu}\right] \mathrm{Lu}-$ PSMA-617 therapy is estimated to be around 10.2 Gy [37]. With a few of such cycles, the cumulative dose to the salivary glands can exceed these limits, and the risk of severe or irreversible xerostomia is greatly increased. Therefore, even limited increases from gustatory stimulation may elicit clinical differences in patients and should be avoided. This is especially applicable to alpha therapy with $\left[{ }^{225} \mathrm{Ac}\right] \mathrm{Ac}-\mathrm{PSMA}-617$, wherein the dose to the glands per cycle is much higher, and for which recommended guidelines are yet to be published.

\section{Conclusion}

In conclusion, eating during the biodistribution phase slightly increased (7-9\%) uptake of $\left[{ }^{18} \mathrm{~F}\right] \mathrm{DCFPyl}$ in the parotid glands. We nonetheless recommend refraining from gustatory stimulation during the administration and early biodistribution phase of radionuclide therapy with PSMA-ligands to reduce the risk of avoidable additional toxicity. Moreover, we recommend that non-pharmacological interventions, such as the one investigated in this study, be tested in a diagnostic setting before application in therapy to prevent unforeseen and undesirable changes in uptake. 


\section{Abbreviations}

${ }^{225}$ Ac: Actinium-225; CT: Computed tomography; ${ }^{18}$ F: Fluorine-18; ${ }^{68} \mathrm{Ga}: \mathrm{Gal}-$ lium-68; ${ }^{131}$ I: lodine-131; ${ }^{177}$ Lu: Lutetium-177; RLT: Radioligand therapy; NIS: Sodium iodide symporter; PET: Positron emission tomography; PSMA: Prostate-specific membrane antigen; SUL: Standardised uptake value corrected for lean body mass; ${ }^{99 \mathrm{~m}} \mathrm{Tc}$ : Technetium-99 m; VOI: Volume of interest.

\section{Supplementary Information}

The online version contains supplementary material available at https://doi. org/10.1186/s13550-021-00838-y.

Additional file 1. Supplementary Material.

\section{Acknowledgements}

Not applicable.

\section{Authors' contributions}

WW and NMB contributed to the study conception and design. NMB contributed to material preparation, patient inclusion and data collection. Analysis was performed by VM. Interpretation of results and supervision of the analysis were done by WVV, J-JS and JBK. The first draft of the manuscript was written by VM. The manuscript was critically read, and feedback was provided by all authors. All authors read and approved the final version.

\section{Funding}

This work was supported by the Dutch Cancer Society KWF [Research Grant: 10606/2016-2]

\section{Availability of data and materials}

The datasets generated and analyzed for this work may be available from the corresponding author on reasonable request.

\section{Declarations}

\section{Ethics approval and consent to participate}

The Medical Ethics Committee of the Netherlands Cancer Institute (CCMO trial registration NL71902.031.20) approved the study protocol. The study was conducted in accordance with the Declaration of HELSINKI. Written and oral informed consent was obtained from all patients prior to study entry.

\section{Consent for publication}

Not applicable.

\section{Competing interests}

The authors have no conflicts of interest to declare that are relevant to the content of this article.

\section{Author details}

${ }^{1}$ Department of Nuclear Medicine, The Netherlands Cancer Institute, Plesmanlaan 121, 1066 CX Amsterdam, The Netherlands. ${ }^{2}$ Department of Radiation Oncology, The Netherlands Cancer Institute, Amsterdam, The Netherlands.

Received: 1 July 2021 Accepted: 11 September 2021

Published online: 26 September 2021

\section{References}

1. Trover JK, Beckett ML, Wright GL. Detection and characterization of the prostate-specific membrane antigen (PSMA) in tissue extracts and body fluids. Int J Cancer. 1995;62:552-8.

2. Israeli RS, Powell CT, Corr JG, Fair WR, Heston WDW. Expression of the prostate-specific membrane antigen1. Cancer Res. 1994;54:1807-11.

3. Wolf P, Freudenberg N, Bühler P, Alt K, Schultze-Seemann W, Wetterauer $U$, et al. Three conformational antibodies specific for different PSMA epitopes are promising diagnostic and therapeutic tools for prostate cancer. Prostate. 2010;70:562-9.
4. Rupp NJ, Umbricht CA, Pizzuto DA, Lenggenhager D, Töpfer A, Müller J, et al. First clinicopathologic evidence of a non-PSMA-related uptake mechanism for 68Ga-PSMA-11 in salivary glands. J Nucl Med. 2019;60:1270-6.

5. Tönnesmann R, Meyer PT, Eder M, Baranski AC. [177 Lu]Lu-PSMA-617 salivary gland uptake characterized by quantitative in vitro autoradiography. Pharmaceuticals. 2019:12:1-9.

6. Rahbar K, Ahmadzadehfar H, Kratochwil C, Haberkorn U, Schafers M, Essler M, et al. German multicenter study investigating 177Lu-PSMA-617 radioligand therapy in advanced prostate cancer patients. J Nucl Med. 2017;58:85-90.

7. Kratochwil C, Bruchertseifer F, Giesel FL, Weis M, Verburg FA, Mottaghy F, et al. 225Ac-PSMA-617 for PSMA-targeted a-radiation therapy of metastatic castration-resistant prostate cancer. J Nucl Med. 2016:57:1941-4.

8. Kratochwil C, Bruchertseifer F, Rathke H, Hohenfellner M, Giesel FL, Haberkorn U, et al. Targeted a-therapy of metastatic castration-resistant prostate cancer with 225 Ac-PSMA-617: Swimmer-plot analysis suggests efficacy regarding duration of tumor control. J Nucl Med. 2018;59:795-802.

9. Luster M, Clarke SE, Dietlein M, Lassmann M, Lind P, Oyen WJG, et al. Guidelines for radioiodine therapy of differentiated thyroid cancer. Eur J Nucl Med Mol Imaging. 2008;35:1941-59.

10. La Perle KMD, Kim DC, Hall NC, Bobbey A, Shen DH, Nagy RS, et al. Modulation of sodium/iodide symporter expression in the salivary gland. Thyroid. 2013;23:1029-36.

11. Mandel SJ, Mandel L. Radioactive iodine and the salivary glands. Thyroid. 2003;13:265-71.

12. Schwenzer NF, Schraml C, Martirosian P, Boss A, Claussen CD, Schick F. MR measurement of blood flow in the parotid gland without contrast medium: a functional study before and after gustatory stimulation. NMR Biomed. 2008;21:598-605

13. Van Nostrand D, Atkins F, Bandaru WV, Chennupati SP, Moreau S, Burman $\mathrm{K}$, et al. Salivary gland protection with sialagogues: a case study. Thyroid. 2009;19:1005-8.

14. James WPT. Research on obesity. Nutr Bull. 1977. https://doi.org/10. 1111/j.1467-3010.1977.tb00966.x.

15. Li X, Rowe SP, Leal JP, Gorin MA, Allaf ME, Ross AE, et al. Semiquantitative parameters in PSMA-targeted PET imaging with 18F-DCFPyL: variability in normal-organ uptake. J Nucl Med. 2017:58:942-6.

16. R Core Team/R Foundation for Statistical Computing. R: a language and environment for statistical computing. Vienna, Austria; 2021. Available from: https://www.r-project.org/.

17. Bates D, Mächler M, Bolker BM, Walker SC. Fitting linear mixed-effects models using Ime4. J Stat Softw. 2015;67.

18. Christou A, Papastavrou E, Merkouris A, Frangos S, Tamana P, Charalambous A. Clinical studies of nonpharmacological methods to minimize salivary gland damage after radioiodine therapy of differentiated thyroid carcinoma: systematic review. Evid Based Comp Altern Med. 2016;2016.

19. Nakada K, Ishibashi T, Takei T, Hirata K, Shinohara K, Katoh S, et al. Does lemon candy decrease salivary gland damage after radioiodine therapy for thyroid cancer? J Nucl Med. 2005;46:261-6.

20. Van Nostrand D, Bandaru V, Chennupati S, Wexler J, Kulkarni K, Atkins F, et al. Radiopharmacokinetics of radioiodine in the parotid glands after the administration of lemon juice. Thyroid. 2010:20:1113-9.

21. Kulkarni K, Van Nostrand D, Atkins F, Mete M, Wexler J, Wartofsky L. Does lemon juice increase radioiodine reaccumulation within the parotid glands more than if lemon juice is not administered? Nucl Med Commun 2014:35:210-6.

22. Jentzen W, Balschuweit D, Schmitz J, Freudenberg L, Eising E, Hilbel T, et al. The influence of saliva flow stimulation on the absorbed radiation dose to the salivary glands during radioiodine therapy of thyroid cancer using 124I PET(/CT) imaging. Eur J Nucl Med Mol Imaging. 2010;37:2298306. https://doi.org/10.1007/s00259-010-1532-z.

23. Liu B, Kuang A, Huang R, Zhao Z, Zeng Y, Wang J, et al. Influence of vitamin $C$ on salivary absorbed dose of $131 \mathrm{l}$ in thyroid cancer patients: a prospective, randomized, single-blind, controlled trial. J Nucl Med. 2010;51:618-23.

24. Jentzen W, Richter M, Nagarajah J, Poeppel TD, Brandau W, Dawes C, et al. Chewing-gum stimulation did not reduce the absorbed dose to salivary glands during radioiodine treatment of thyroid cancer as inferred from pre-therapy 124I PET/CT imaging. EJNMMI Phys. 2014;1:1-11. 
25. Ghosh A, Heston WDW. Tumor target prostate specific membrane antigen (PSMA) and its regulation in prostate cancer. J Cell Biochem. 2004;91:528-39.

26. Langbein T, Chaussé G, Baum RP. Salivary gland toxicity of PSMA radioligand therapy: relevance and preventive strategies. J Nucl Med. 2018;59:1172-3.

27. Edgar WM. Saliva and dental health. Clinical implications of saliva: report of a consensus meeting. Br Dent J. 1990;169:96-8.

28. Afshar-Oromieh A, Avtzi E, Giesel FL, Holland-Letz T, Linhart HG, Eder M, et al. The diagnostic value of PET/CT imaging with the 68Ga-labelled PSMA ligand HBED-CC in the diagnosis of recurrent prostate cancer. Eur J Nucl Med Mol Imaging. 2015;42:197-209.

29. olde Heuvel J, de Wit-van der Veen BJ, Sinaasappel M, Slump CH, Stokkel MPM. Early differences in dynamic uptake of 68Ga-PSMA-11 in primary prostate cancer: a test-retest study. PLoS ONE. 2021;16:6394. https://doi. org/10.1371/journal.pone.0246394.

30. Zechmann CM, Afshar-Oromieh A, Armor T, Stubbs JB, Mier W, Hadaschik B, et al. Radiation dosimetry and first therapy results with a 124I/ 1311-labeled small molecule (MIP-1095) targeting PSMA for prostate cancer therapy. Eur J Nucl Med Mol Imaging. 2014:41:1280-92.

31. Afshar-Oromieh A, Haberkorn U, Zechmann C, Armor T, Mier W, Spohn $F$, et al. Repeated PSMA-targeting radioligand therapy of metastatic prostate cancer with 131I-MIP-1095. Eur J Nucl Med Mol Imaging. 2017:44:950-9.

32. van Kalmthout LWM, Lam MGEH, de Keizer B, Krijger GC, Ververs TFT, de Roos R, et al. Impact of external cooling with icepacks on 68Ga-PSMA uptake in salivary glands. EJNMMI Res. 2018;8.
33. Yilmaz B, Nisli S, Ergul N, Gursu RU, Acikgoz O, Cxermik TF. Effect of external cooling on 177Lu-PSMA uptake by the parotid glands. J Nucl Med. 2019:60:1388-93.

34. Kratochwil C, Fendler WP, Eiber M, Baum R, Bozkurt MF, Czernin J, et al. EANM procedure guidelines for radionuclide therapy with $177 \mathrm{Lu}-$ labelled PSMA-ligands (177Lu-PSMA-RLT). Eur J Nucl Med Mol Imaging. 2019;46:2536-44. https://doi.org/10.1007/s00259-019-04485-3.

35. Deasy JO, Moiseenko V, Marks L, Chao KSC, Nam J, Eisbruch A. Radiotherapy dose-volume effects on salivary gland function. Int J Radiat Oncol Biol Phys. 2010;76:S58-63.

36. Murdoch-Kinch CA, Kim HM, Vineberg KA, Ship JA, Eisbruch A. Doseeffect relationships for the submandibular salivary glands and implications for their sparing by intensity modulated radiotherapy. Int J Radiat Oncol Biol Phys. 2008;72:373-82.

37. Kratochwil C, Bruchertseifer F, Rathke H, Bronzel M, Apostolidis C, Weichert W, et al. Targeted a-therapy of metastatic castration-resistant prostate cancer with 225Ac-PSMA-617: dosimetry estimate and empiric dose finding. J Nucl Med. 2017;58:1624-31. https://doi.org/10.2967/ jnumed.117.191395.

\section{Publisher's Note}

Springer Nature remains neutral with regard to jurisdictional claims in published maps and institutional affiliations.

\section{Submit your manuscript to a SpringerOpen ${ }^{\circ}$ journal and benefit from:}

- Convenient online submission

- Rigorous peer review

- Open access: articles freely available online

- High visibility within the field

- Retaining the copyright to your article

Submit your next manuscript at $\boldsymbol{\nabla}$ springeropen.com 